\title{
Dosimetric impacts of applicator displacements and applicator reconstruction-uncertainties on 3D image-guided brachytherapy for cervical cancer
}

\author{
Joshua Schindel, BS!, Winson Zhang!' Sudershan K. Bhatia, MD, PhD, MPH², Wenging Sun, MD, PhD², Yusung Kim, PhD² \\ 'Biomedical Engineering Department, ${ }^{2}$ Radiation Oncology Department, The University of lowa, lowa City, USA
}

\begin{abstract}
Purpose: To quantify the dosimetric impact of applicator displacements and applicator reconstruction-uncertainties through simulated planning studies of virtual applicator shifts.

Material and methods: Twenty randomly selected high-dose-rate (HDR) titanium tandem-and-ovoid (T\&O) plans were retrospectively studied. MRI-guided, conformal brachytherapy (MRIG-CBT) plans were retrospectively generated. To simulate $\mathrm{T} \& \mathrm{O}$ displacement, the whole $\mathrm{T} \& \mathrm{O}$ set was virtually shifted on treatment planning system in the cranial (+) and the caudal (-) direction after each dose calculation. Each shifted plan was compared to an unshifted plan. To simulate $\mathrm{T} \& \mathrm{O}$ reconstruction-uncertainties, each tandem and ovoid was separately shifted along its axis before performing the dose calculation. After the dose calculation, the calculated isodose lines and T\&O were moved back to unshifted T\&O position. Shifted and shifted-back plan were compared.

Results: Regarding the dosimetric impact of the simulated T\&O displacements, rectal $\mathrm{D}_{2 \mathrm{cc}}$ values were observed as being the most sensitive to change due to $\mathrm{T} \& \mathrm{O}$ displacement among all dosimetric metrics regardless of point $\mathrm{A}$ $(p<0.013)$ or MRIG-CBT plans $(p<0.0277)$. To avoid more than $10 \%$ change, $\pm 1.5 \mathrm{~mm}$ T\&O displacements were accommodated for both point A and MRIG-CBT plans. The dosimetric impact of T\&O displacements on sigmoid $(p<0.0005)$, bladder $(p<0.0001)$, HR-CTV $(p<0.0036)$, and point A $(p<0.0015)$ were significantly larger in the MRIG-CBT plans than point A plans. Regarding the dosimetric impact of T\&O reconstruction-uncertainties, less than $\pm 3.0 \mathrm{~mm}$ reconstruction-uncertainties were also required in order to avoid more than $10 \%$ dosimetric change in either the point $\mathrm{A}$ or MRIG-CBT plans.

Conclusions: The dosimetric impact of simulated T\&O displacements was significantly larger in the MRIG-CBT plans than in the point A plans. Either $\pm 3 \mathrm{~mm}$ T\&O displacement or a $\pm 4.5 \mathrm{~mm}$ T\&O reconstruction-uncertainty could cause greater than $10 \%$ dosimetric change for both point A plans and MRIG-CBT plans.
\end{abstract}

J Contemp Brachytherapy 2013; 5, 4: 250-257 DOI: $10.5114 /$ jcb.2013.39453

Key words: 3D image, applicator shifts, brachytherapy, cervical cancer, dosimetry, high-dose-rate.

\section{Purpose}

3D image-based treatment planning for gynaecological (GYN) cancer is a recent innovation [1-7] where magnetic resonance imaging (MRI) and computerized tomography (CT) are used to accurately define target volumes, organs at risk (OARs), and applicator position [5]. Dose calculations are dependent on the geometrical accuracy of the source position relative to target volumes and OARs [8]. The challenge with $3 \mathrm{D}$ image guidance in most centers is that the patient must be transferred out of the highdose-rate (HDR) brachytherapy (BT) operating room for imaging. The patient transfer between applicator implantation, imaging, and radiation delivery can cause applicator displacements. These applicator displacements lead to dosimetric errors to the target volume and OARs $[4,8]$. Gerzten et al. have researched applicator shifts due to patient movement from simulation to HDR treatment rooms [9]. However, applicator displacements were evaluated on orthogonal radiographs, and no dosimetric impact on target volumes and OARs were measured. Real time applicator monitoring systems using Microsoft's KINECT ${ }^{\mathrm{TM}}$ system (Microsoft Corp. Redmond, WA, USA) was recently introduced as a possible solution to monitor intracavitary applicator displacement with less than or equal to $1 \mathrm{~mm}$ resolution for applicator displacement during additional 3D scans in a different room [10]. However, to set applicator displacement limits, it is important to first understand its dosimetric impact. No study has yet presented the dosimetric impact of intracavitary applicator
Address for correspondence: Yusung Kim, PhD, DABR, Asst. Prof., Radiation Oncology Department, The University of Iowa, 200 Hawkins Drive, 01607 PFP-W, Iowa City, IOWA 52242-1009, USA, phone: +1 319-384-9406, ® e-mail: yusung-kim@uiowa.edu
Received: 20.08.2013

Accepted: 26.11.2013

Published: 31.12.2013 
displacements for both conventional, point A plans and 3D image-guided, conformal plans.

Another major challenge facing 3D image guided planning is to reconstruct the source-pathway on the 3D image, especially those on MRI. Exclusive MRI-based applicator reconstruction methodologies were introduced for different applicators for cervical cancer such as plastic tandem-and-ovoids (T\&O) [11,12], plastic tandem-and-ring (T\&R) applicators [13], titanium T\&R applicators [13], titanium Rotterdam applicators [14], and Vienna applicators (plastic T\&R plus supplementary needles) [3]. Among the first applicator reconstruction accuracy studies $[4,6,11-15]$ on MRI, two reports $[7,14]$ presented absolute reconstruction uncertainties using phantom studies for plastic and titanium T\&R [7] and titanium Rotterdam applicators [14], while other reports presented relative comparison data using different reconstruction methods [15] or different MRI scanning protocols [12]. When a plastic applicator is used for MRI guided BT, the signals of the MRI-marker catheters [11] are able to guide clinicians to reconstruct applicator position based on their hyposignals. The use of titanium applicators using MRI makes their reconstruction much more difficult due to the artifacts they create. Recently a novel Marker-Flange [16] was introduced to improve the reconstruction accuracy of titanium applicators when used with applicator library. GEC-ESTRO (Groupe Européen de Curiethérapie-European Society for Therapeutic Radiology and Oncology) reported the considerations and pitfalls in new applicator commissioning, and the reconstruction uncertainties this entailed with a 3D image [4]. However, 3D imaging reconstruction uncertainties should always be validated in each clinic when a clinic implements a 3D image guided BT program, since reconstruction uncertainties are associated with each institutional scanning protocols, the type of CT or MRI scanner, the type of applicator, applicator material, treatment planning system (TPS), and planning workflow. For instance, when a titanium applicator is used with a CT scanner, the titanium applicator on the CT image can have a bigger diameter (e.g. $6 \mathrm{~mm}$ versus $3.2 \mathrm{~mm}$ ) due to the Hounsfield unit changes between the titanium and adjacent tissue. Namely, when a default-window level is used, if a clinician defines the end of bright white signal on CT as a tip of tandem, this can cause systematic reconstruction errors that result in systematic dosimetric errors. It is important to understand the estimated dosimetric impact of the reconstruction uncertainties. For MRI-guided, conformal BT plans, Tanderup et al. [8] presented the dosimetric impact of $T \& R$ on target volumes and OARs through by virtually shifting or rotating each tandem and ring before performing dose calculations of volume optimization. No study has yet presented the dosimetric impact of reconstruction accuracy for conventional, point A plans. In this study, we present the dosimetric impact of applicator displacement and applicator reconstruction-uncertainties on target volumes and OARs for both conventional, point-A plans, and 3D image guided conformal plans.

\section{Material and methods \\ HDR brachytherapy planning: point $A$ and MRIG-CBT plans}

Conventional point A plans were clinically generated on for MRI, following the recommendations of the American Brachytherapy Society consensus guidelines $[17,18]$. The BrachyVision ${ }^{\mathrm{TM}}$ (Varian Medical Systems Inc., Palo Alto, CA, USA) TPS was used. A high resolution (3.0 Tesla) Siemens MAGNETOM Trio 3T MR scanner (Siemens Medical Solutions Inc., Erlangen, Germany) was used for all MR scanning following the same GYN MRI protocols as previously reported [19]. Twenty randomly selected HDR cases were retrospectively studied after Institutional Review Board approval was secured. A titanium Fletcher-Suit-Delclos T\&O applicator (Varian Medical Systems, Inc.) was used for all implants, along with gauze packing. The position of the bladder foley at the most inferior bladder neck was checked after foley insertion, and also re-checked on MRI. Orthogonal radiographs using a $\mathrm{C}$-arm were taken before and after each MRI scan (Pre- and Post-X-ray). The institutional protocols for external beam radiotherapy and HDR BT fractionation schemes were detailed in previous studies [7,20-22]. High-risk clinical target volumes (HR-CTV), of the rectum, bladder, and sigmoid were retrospectively delineated as per GEC-ESTRO recommendations [2,3]. For each point A plan, a MRI-guided, conformal BT (MRIGCBT) plan was retrospectively generated using the hybrid-inverse optimization detailed in a previous study [7].

\section{Simulating $T \& O$ displacements and T\&O reconstruction-uncertainties}

To simulate $\mathrm{T} \& \mathrm{O}$ displacements, in unshifted, original point A plans (Figs. 1B,D) and MRIG-CBT (Figs. 1C,E), a whole T\&O (Fig. 1A) was virtually shifted in a cranial $(+)$ or caudal (-) direction in $\pm 1.5, \pm 3, \pm 5, \pm 6, \pm 7.5, \pm 10$, and $\pm 20 \mathrm{~mm}$ increments after dose calculations (Fig. 1). Simply put, the whole $\mathrm{T} \& \mathrm{O}$ was shifted cranio-caudally while dwell times and positions were kept unchanged (the unshifted plan) in order to model the physical $\mathrm{T} \& \mathrm{O}$ displacement that can occur during patient transfer between the BT treatment room and CT or MRI scanning room. Given that, the $\mathrm{T} \& \mathrm{O}$ is tightly packed with gauze and tandem angle limits torque, the T\&O rarely rotates, but is often displaced in a cranial-caudal direction during 3D scanning. For the purpose of this study, only the $\mathrm{T} \& \mathrm{O}$ displacement in cranial-caudal direction was simulated in this study. Absolute dosimetric changes due to virtual shifting were measured for both point-A and MRIG-CBT plans between an unshifted and a shifted plan.

Regarding T\&O reconstruction-uncertainty simulations, a tandem and two ovoids were shifted along their central axis (see Fig. 2A), since reconstruction uncertainties exist mainly along their central axis when an applicator library is used. Unlike that the ring reconstruction uncertainties for T\&R that occur when it rotates, ovoid reconstruction uncertainties for T\&O occur in a posterior-anterior direction along the central axis of the ovoid-tandem 

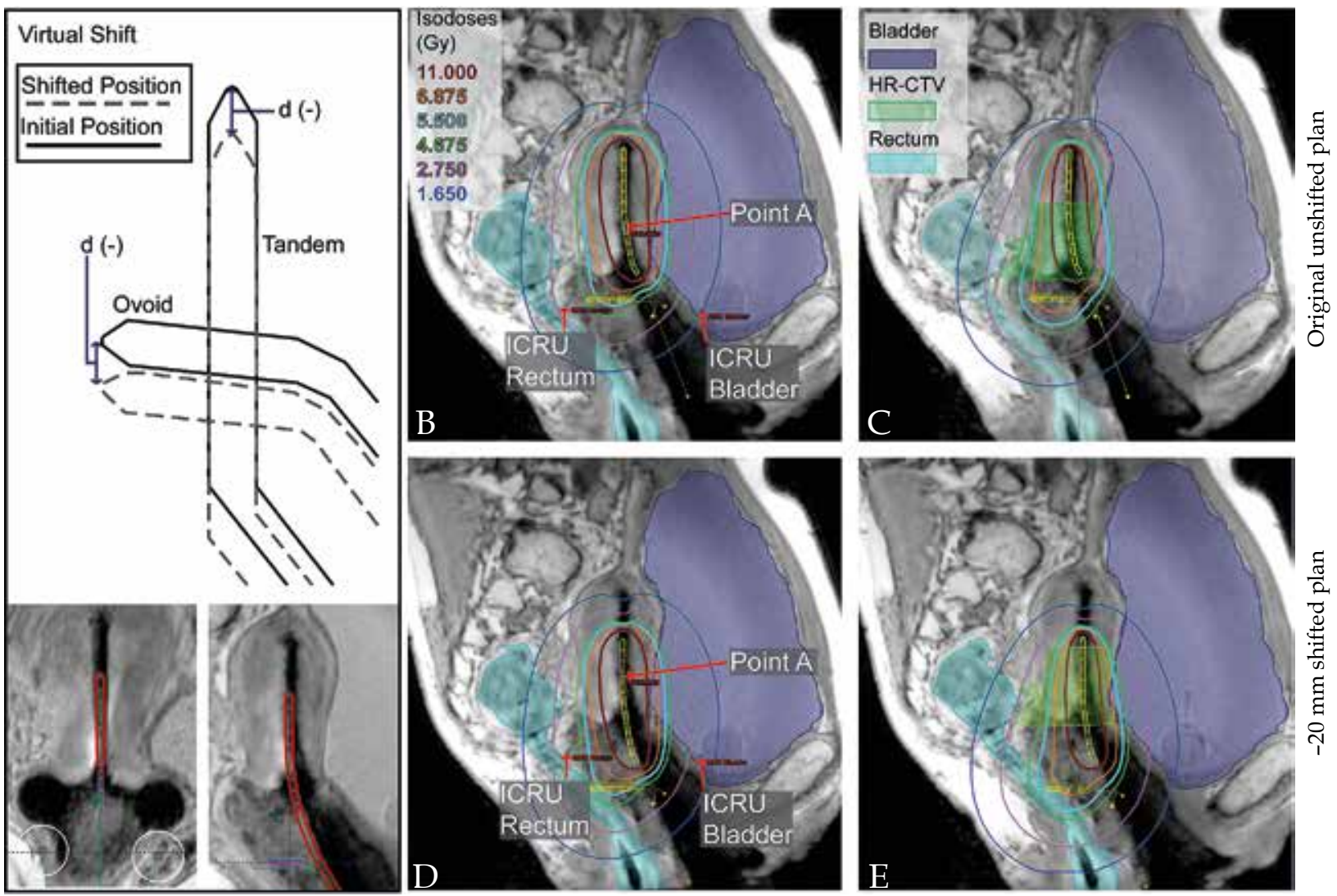

Fig. 1. The simulated $T \& O$ displacements on a treatment planning system by virtual shifting whole $T \& O$ (A). The original unshifted plans of conventional, point A technique (B) and MRIG-CBT (C). The resulting isodose lines due to $-20 \mathrm{~mm}$ shift for conventional plan (D) and MRIG-CBT plan (E)

(Fig. 2A). We defined (+) direction and (-) direction for either tandem or ovoid motion when a source-wire travels longer (shorter), along its source-pathway. As a result, a tandem was virtually shifted in a cranial (+) or caudal $(-)$ direction, while each ovoid was virtually shifted in a posterior $(+)$ or anterior $(-)$ direction. For the purpose of this study, a tandem and both ovoids were virtually shifted in increments of $\pm 1.5, \pm 3.0, \pm 4.5, \pm 6.0, \pm 7.5$, and $\pm 10 \mathrm{~mm}$. For instance, a $-10 \mathrm{~mm}$ shifted plan represents a tandem is shifted $10 \mathrm{~mm}$ in a caudal direction and each ovoid is shifted $10 \mathrm{~mm}$ in an anterior direction as depicted in Fig. 2A. After virtual shifting was completed, dose calculations of either a point A plan or a MRIG-CBT plan was performed, given applicator reconstruction. For the point A plan (Fig. 2B), reference optimization lines were created based upon the shifted applicator and optimization was done using those regenerated reference lines. For the MRIG-CBT plan (Fig. 2C), a hybrid-inverse optimization $[7,23]$ was performed after each shift. It is noted that the isodose lines (i.e. dwell times) are delivered at the correct dwell positions, even when an applicator is reconstructed inaccurately (i.e. a shift of a couple of $\mathrm{mm}$ from the true position). To simulate delivered-isodose lines, we shifted the calculated isodose lines and the T\&O back to its original position (Figs. $2 \mathrm{D}, \mathrm{E}$ ) which we then used as a reference plan. Each shifted plan has its own reference plan. Afterward, we compared the dose parameters between the shifted plans (Figs. 2 B,C) and their reference plans (Figs. 2 D,E).

Dosimetric changes on dose-volume parameters of the HR-CTV $\left(D_{100}\right.$ and $\left.D_{90}\right)$, the rectum $\left(D_{2 c c}\right)$, the bladder $\left(D_{2 c c}\right)$, and the sigmoid $\left(D_{2 c c}\right)$ were recorded for both point $\mathrm{A}$ and MRIG-CBT plans. In addition, dosimetric changes of point A (left and right points) and ICRU report \#38 defined, rectal and bladder points were quantified. To normalize the prescription dose differences, the percent dose differences were calculated for all dosimetric metrics. The $p$-values were calculated using a two-tailed $t$-test.

\section{Results}

\section{Dosimetric impact of T\&O displacements}

The dosimetric impact of simulated T\&O displacements on sigmoid $(p<0.0005)$, bladder $(p<0.0001)$, HR-CTV $(p<0.0036)$, and point $\mathrm{A}(p<0.0015)$ were significantly larger for the MRIG-CBT plans than the point A plans. MRIG-CBT plans data in Table 1 clearly presented more dark (dosimetric changes $\geq 15 \%$ ) and gray (dosimetric changes $\geq 10 \%$ ) regions than point A plans as seen in Table 2 . Rectal $D_{2 c c}$ values were observed as the most sensitive to change due to the T\&O dis- 
A

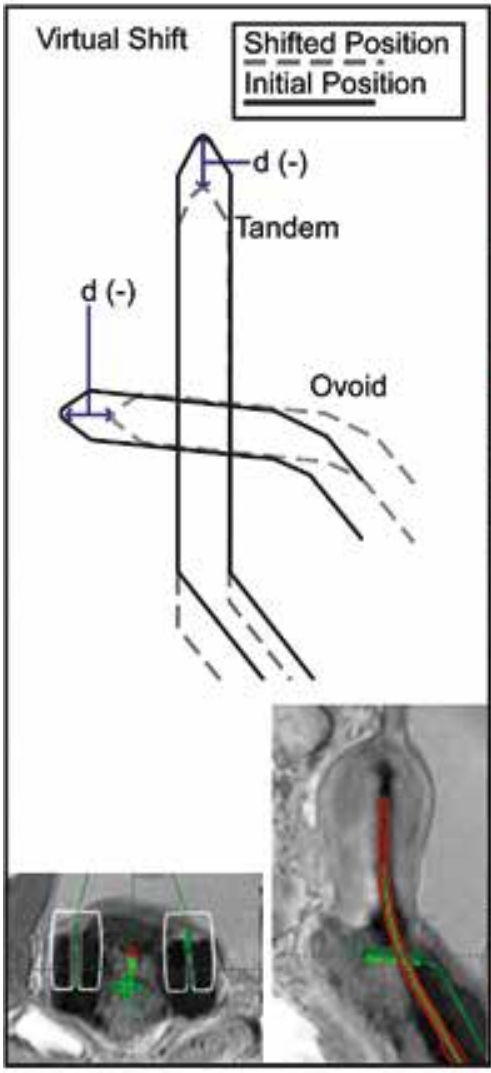

Conventional Plan

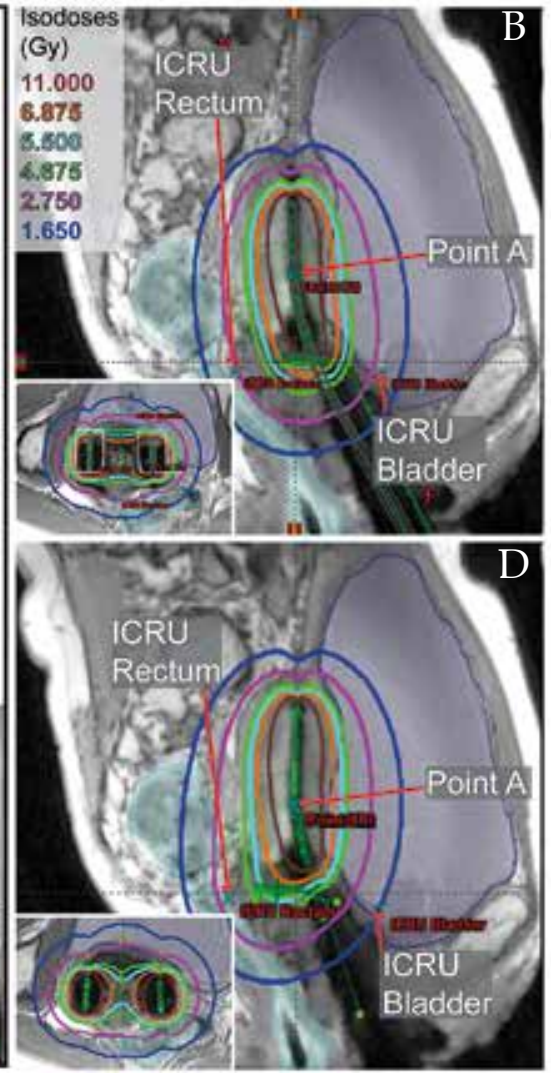

Conformal Plan

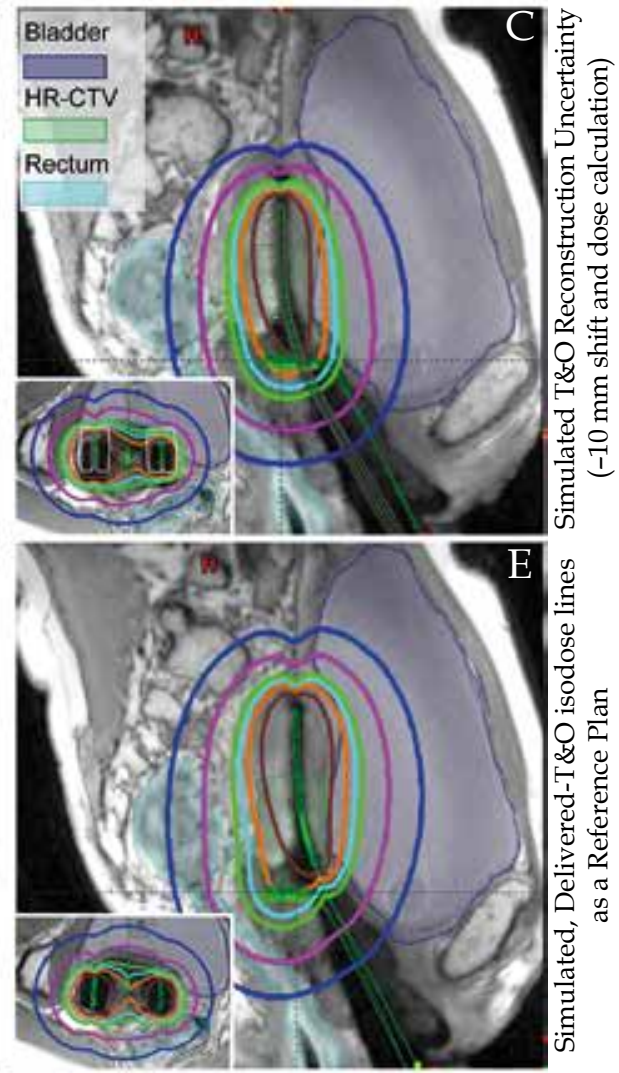

Fig. 2. The simulated T\&O reconstruction uncertainties on a treatment planning system by shifting each tandem and ovoid (A). The resulting isodose lines of conventional plan (B) and MRIG-CBT plan (C) after $-10 \mathrm{~mm}$ shift to simulate T\&O reconstruction uncertainties and dose calculation. To evaluate their dosimetric impacts, the plans of B and $\mathrm{C}$ were compared to their simulated, delivered plans (D and E, respectively) that were created by shifting T\&O back to their original position and dose calculation

placement across all dosimetric metrics, regardless of point $\mathrm{A}$ $(p<0.013)$ or MRIG-CBT plans $(p<0.0277)$. Even a $3 \mathrm{~mm}$ $\mathrm{T} \& \mathrm{O}$ displacement caused an average $10-12 \%$ dosimetric change on rectal $\mathrm{D}_{2 \mathrm{cc}}$ for both point $\mathrm{A}$ plans (Table 2) and MRIG-CBT plans (Table 1). A $5 \mathrm{~mm}$ T\&O displacement resulted in clinically unacceptable dosimetric changes on rectal $D_{2 \mathrm{cc}}$ for point A plans (average $17-21 \%$, Table 2) and MRIG-CBT plans (average 16-22\%, Table 1). A $1.5 \mathrm{~mm}$ $\mathrm{T} \& \mathrm{O}$ displacement solely presented less than a $10 \%$ dosimetric change (average 6\%) for both point A (Table 2) and MRIG-CBT plans (Table 1 ). The $\mathrm{D}_{2 \mathrm{cc}}$ value for rectum showed a larger dosimetric change than the ICRU rectal point doses for point A $(p<0.0389)$ and MRIG-CBT plans $(p<0.0699)$. A more than $5 \mathrm{~mm}$ T\&O displacement in a cranial direction resulted in a greater than $10 \%$ change in bladder $\mathrm{D}_{2 \mathrm{cc}}$ for MRIG-CBT plans. Bladder $\left(\mathrm{D}_{2 \mathrm{cc}}\right)$ of point A plans presented a relatively low dosimetric change (less than 10\%) up to $7.5 \mathrm{~mm} \mathrm{T \& O}$ displacement. Sigmoid $\left(D_{2 c c}\right)$ also had a relatively low dosimetric change (less than $10 \%$ ) up to $10 \mathrm{~mm}$ and $7.5 \mathrm{~mm}$ T\&O displacement for both point A and MRIG-CBT plans, respectively. HR-CTV $\left(D_{100}\right.$ and $\left.D_{90}\right)$ was also found to change less than $10 \%$ for up to $10 \mathrm{~mm}(7.5 \mathrm{~mm})$ of the T\&O displacement for MRIG-CBT plans (point A plans). The point A doses were found to be less than $10 \%$ of the changes for up to $10 \mathrm{~mm}$ in the $\mathrm{T} \& \mathrm{O}$ displacement for point A plans, since point A locates on the reference optimization lines. However, more changes in the point A doses were observed (11-17\% changes per $10 \mathrm{~mm}$ displacement) in MRIG-CBT plans, since they don't have typical pear-shape isodose lines, but have conformal isodose lines.

\section{Dosimetric impact of $\mathrm{T} \& \mathrm{O}$ reconstruction- uncertainties}

Our findings suggest that in order to avoid more than $10 \%$ dosimetric changes in any dosimetric parameter either point A (Table 4) or MRIG-CBT plans (Table 3), had to have less than or equal to $\pm 3.0 \mathrm{~mm}$ reconstruction-uncertainty. For conventional point A plans, the rectum $\left(\mathrm{D}_{2 \mathrm{c} c^{\prime}}\right.$ ICRU point) and bladder (ICRU point) presented greater dosimetric changes due to the reconstruction uncertainties (Table 4). The rectal $\mathrm{D}_{2 \mathrm{cc}}$ value recorded was the most sensitive to the $\mathrm{T} \& \mathrm{O}$ reconstruction. To ensure that less than $10 \%$ dosimetric change occurred in rectal $\mathrm{D}_{2 \mathrm{cc}}$ a value of less than or equal to $\pm 1.5 \mathrm{~mm}$ in reconstruction uncertainty was required. A $3 \mathrm{~mm}$ reconstruction uncertainty in the cranial direction caused an average $15 \%$ dosimetric change in rectal $\mathrm{D}_{2 \mathrm{cc}}$. ICRU bladder point presented a greater than 
Table 1. The simulated dosimetric impacts of T\&O displacements for MRIG-CBT (20 plans) by virtually shifting whole $T \& O$ in a treatment planning system

\begin{tabular}{|c|c|c|c|c|c|c|c|c|c|c|c|c|c|c|}
\hline & \multicolumn{14}{|c|}{ Simulated dosimetric impacts [\%] of T\&O displacements for MRIG-CBT plans } \\
\hline & \multicolumn{6}{|c|}{ Caudal T\&O shift } & \multicolumn{8}{|c|}{ Cranial T\&O shift } \\
\hline & $\begin{array}{l}-20 \\
\mathrm{~mm}\end{array}$ & $\begin{array}{l}-10 \\
\mathrm{~mm}\end{array}$ & $\begin{array}{c}-7.5 \\
\mathrm{~mm}\end{array}$ & $\begin{array}{c}-6 \\
\mathrm{~mm}\end{array}$ & $\begin{array}{c}-5 \\
\mathrm{~mm}\end{array}$ & $\begin{array}{c}-3 \\
\mathrm{~mm}\end{array}$ & $\begin{array}{c}-1.5 \\
\mathrm{~mm}\end{array}$ & $\begin{array}{c}1.5 \\
\mathrm{~mm}\end{array}$ & $\begin{array}{c}3 \\
\mathrm{~mm}\end{array}$ & $\begin{array}{c}5 \\
\mathrm{~mm}\end{array}$ & $\begin{array}{c}6 \\
\mathrm{~mm}\end{array}$ & $\begin{array}{l}7.5 \\
\mathrm{~mm} \\
\end{array}$ & $\begin{array}{c}10 \\
\mathrm{~mm}\end{array}$ & $\begin{array}{c}20 \\
\mathrm{~mm}\end{array}$ \\
\hline HR-CTV D $90[\%]$ & $23 \pm 11$ & $9 \pm 5$ & $7 \pm 4$ & $9 \pm 3$ & $5 \pm 3$ & $3 \pm 2$ & $2 \pm 1$ & $1 \pm 1$ & $3 \pm 2$ & $5 \pm 4$ & $7 \pm 5$ & $8 \pm 6$ & $11 \pm 8$ & $21 \pm 16$ \\
\hline HR-CTV D $100[\%]$ & $20 \pm 9$ & $6 \pm 9$ & $7 \pm 5$ & $6 \pm 4$ & $5 \pm 4$ & $3 \pm 3$ & $2 \pm 2$ & $2 \pm 1$ & $4 \pm 2$ & $6 \pm 3$ & $7 \pm 4$ & $9 \pm 5$ & $11 \pm 7$ & $24 \pm 11$ \\
\hline Rectum $D_{2 c c}[\%]$ & $136 \pm 46$ & $50 \pm 13$ & $35 \pm 8$ & $27 \pm 6$ & $22 \pm 5$ & $12 \pm 3$ & $6 \pm 1$ & $6 \pm 1$ & $10 \pm 2$ & $16 \pm 4$ & $19 \pm 4$ & $23 \pm 5$ & $29 \pm 6$ & $47 \pm 10$ \\
\hline Badder $\mathrm{D}_{2 c c}[\%]$ & $29 \pm 10$ & $14 \pm 7$ & $11 \pm 5$ & $8 \pm 5$ & $7 \pm 4$ & $4 \pm 3$ & $2 \pm 1$ & $2 \pm 2$ & $5 \pm 3$ & $8 \pm 6$ & $10 \pm 7$ & $13 \pm 9$ & $19 \pm 13$ & $50 \pm 42$ \\
\hline Sigmoid $\mathrm{D}_{2 c \mathrm{c}}[\%]$ & $24 \pm 12$ & $12 \pm 6$ & $8 \pm 5$ & $7 \pm 4$ & $5 \pm 3$ & $3 \pm 2$ & $2 \pm 1$ & $1 \pm 1$ & $3 \pm 2$ & $5 \pm 3$ & $5 \pm 3$ & $6 \pm 4$ & $9 \pm 7$ & $13 \pm 9$ \\
\hline Point A left [\%] & $28 \pm 14$ & $12 \pm 8$ & $9 \pm 6$ & $7 \pm 4$ & $6 \pm 4$ & $4 \pm 2$ & $2 \pm 1$ & $2 \pm 1$ & $3 \pm 2$ & $5 \pm 4$ & $7 \pm 4$ & $8 \pm 5$ & $11 \pm 8$ & $41 \pm 31$ \\
\hline Point A right [\%] & $28 \pm 17$ & $13 \pm 9$ & $10 \pm 7$ & $8 \pm 5$ & $7 \pm 4.5$ & $4 \pm 3$ & $2 \pm 2$ & $2 \pm 2$ & $4 \pm 3$ & $8 \pm 5$ & $9 \pm 7$ & $12 \pm 9$ & $17 \pm 13$ & $65 \pm 53$ \\
\hline ICRU rectum [\%] & $60 \pm 53$ & $31 \pm 23$ & $23 \pm 16$ & $18 \pm 12$ & $15 \pm 10$ & $9 \pm 6$ & $4 \pm 3$ & $4 \pm 2$ & $8 \pm 4$ & $14 \pm 6$ & $16 \pm 7$ & $20 \pm 8$ & $26 \pm 9$ & $46 \pm 11$ \\
\hline ICRU bladder [\%] & $27 \pm 14$ & $14 \pm 9$ & $11 \pm 6$ & $9 \pm 5$ & $7 \pm 4$ & $5 \pm 3$ & $2 \pm 1$ & $3 \pm 1$ & $5 \pm 3$ & $9 \pm 5$ & $10 \pm 6$ & $13 \pm 8$ & $18 \pm 11$ & $35 \pm 26$ \\
\hline
\end{tabular}

$$
<5 \% \quad \geq 5 \% \quad \geq 10 \% \quad \geq 15
$$

$T \& O$ - tandem-and-ovoids, HR-CTV - high risk clinical target volume, $D_{90}\left(D_{100}\right)$ - minimum dose covering $90 \%(100 \%)$ of the volume, $D_{2 c c}-$ dose of the $2 c c$ of the highest dose, ICRU rectum (bladder) - ICRU report \#38 defined rectal (bladder) point

Table 2. The simulated dosimetric impacts of T\&O displacements for conventional planning (20 plans) by virtually shifting whole $T \& O$ in a treatment planning system

\begin{tabular}{|c|c|c|c|c|c|c|c|c|c|c|c|c|c|c|}
\hline & \multicolumn{14}{|c|}{ Simulated dosimetric impacts [\%] of T\&O displacements for conventional point A system } \\
\hline & \multicolumn{6}{|c|}{ Caudal T\&O shift } & \multicolumn{8}{|c|}{ Cranial T\&O shift } \\
\hline & $\begin{array}{l}-20 \\
\mathrm{~mm}\end{array}$ & $\begin{array}{l}-10 \\
\mathrm{~mm}\end{array}$ & $\begin{array}{l}-7.5 \\
\mathrm{~mm}\end{array}$ & $\begin{array}{c}-6 \\
\mathrm{~mm}\end{array}$ & $\begin{array}{c}-5 \\
\mathrm{~mm}\end{array}$ & $\begin{array}{c}-3 \\
\mathrm{~mm}\end{array}$ & $\begin{array}{l}-1.5 \\
\mathrm{~mm}\end{array}$ & $\begin{array}{c}1.5 \\
\mathrm{~mm}\end{array}$ & $\begin{array}{c}3 \\
\mathrm{~mm}\end{array}$ & $\begin{array}{c}5 \\
\mathrm{~mm}\end{array}$ & $\begin{array}{c}6 \\
\mathrm{~mm} \\
\end{array}$ & $\begin{array}{c}7.5 \\
\mathrm{~mm} \\
\end{array}$ & $\begin{array}{c}10 \\
\mathrm{~mm}\end{array}$ & $\begin{array}{c}20 \\
\mathrm{~mm}\end{array}$ \\
\hline HR-CTV D ${ }_{90}[\%]$ & $9 \pm 7$ & $5 \pm 4$ & $4 \pm 4$ & $3 \pm 3$ & $3 \pm 2$ & $2 \pm 2$ & $1 \pm 1$ & $1 \pm 1$ & $2 \pm 2$ & $3 \pm 3$ & $4 \pm 3$ & $5 \pm 4$ & $7 \pm 5$ & $18 \pm 10$ \\
\hline HR-CTV D $100[\%]$ & $11 \pm 9$ & $6 \pm 4$ & $5 \pm 4$ & $4 \pm 3$ & $3 \pm 2$ & $2 \pm 2$ & $1 \pm 1$ & $2 \pm 2$ & $3 \pm 2$ & $4 \pm 3$ & $5 \pm 4$ & $6 \pm 5$ & $8 \pm 7$ & $18 \pm 10$ \\
\hline Rectum $D_{2 c c}[\%]$ & $138 \pm 60$ & $52 \pm 20$ & $35 \pm 12$ & $27 \pm 8$ & $21 \pm 6$ & $12 \pm 3$ & $6 \pm 1$ & $6 \pm 1$ & $10 \pm 3$ & $17 \pm 4$ & $20 \pm 5$ & $24 \pm 5$ & $29 \pm 6$ & $47 \pm 9$ \\
\hline Badder $\mathrm{D}_{2 c c}[\%]$ & $13 \pm 6$ & $6 \pm 3$ & $5 \pm 2$ & $4 \pm 2$ & $3 \pm 2$ & $2 \pm 1$ & $1 \pm 1$ & $1 \pm 1$ & $2 \pm 1$ & $4 \pm 2$ & $6 \pm 3$ & $8 \pm 4$ & $12 \pm 5$ & $41 \pm 27$ \\
\hline Sigmoid $\mathrm{D}_{2 c c}[\%]$ & $15 \pm 10$ & $6 \pm 5$ & $5 \pm 4$ & $4 \pm 3$ & $3 \pm 2$ & $2 \pm 1$ & $1 \pm 1$ & $1 \pm 1$ & $2 \pm 1$ & $3 \pm 2$ & $3 \pm 3$ & $4 \pm 3$ & $5 \pm 5$ & $11 \pm 8$ \\
\hline Point A left [\%] & $8 \pm 8$ & $3 \pm 2$ & $3 \pm 2$ & $2 \pm 1$ & $2 \pm 1$ & $1 \pm 1$ & $0 \pm 0$ & $0 \pm 1$ & $1 \pm 1$ & $2 \pm 2$ & $2 \pm 2$ & $2 \pm 3$ & $4 \pm 4$ & $41 \pm 26$ \\
\hline Point A right [\%] & $8 \pm 8$ & $3 \pm 3$ & $2 \pm 2$ & $2 \pm 2$ & $2 \pm 2$ & $1 \pm 1$ & $1 \pm 1$ & $1 \pm 1$ & $1 \pm 1$ & $2 \pm 2$ & $3 \pm 2$ & $4 \pm 3$ & $6 \pm 5$ & $55 \pm 61$ \\
\hline ICRU rectum [\%] & $47 \pm 28$ & $25 \pm 14$ & $18 \pm 10$ & $14 \pm 8$ & $12 \pm 6$ & $7 \pm 3$ & $3 \pm 2$ & $4 \pm 2$ & $7 \pm 3$ & $11 \pm 4$ & $14 \pm 5$ & $17 \pm 6$ & $22 \pm 7$ & $42 \pm 9$ \\
\hline ICRU bladder [\%] & $12 \pm 7$ & $6 \pm 4$ & $5 \pm 3$ & $4 \pm 2$ & $3 \pm 2$ & $2 \pm 1$ & $1 \pm 1$ & $1 \pm 1$ & $2 \pm 2$ & $4 \pm 3$ & $5 \pm 3$ & $7 \pm 5$ & $10 \pm 7$ & $24 \pm 17$ \\
\hline
\end{tabular}

$T \& O$-tandem-and-ovoids, HR-CTV - high risk clinical target volume, $D_{90}\left(D_{100}\right)$ - minimum dose covering $90 \%(100 \%)$ of the volume, $D_{2 c c}-$ dose of the $2 c c$ of the highest dose, ICRU rectum (bladder) - ICRU report \#38 defined rectal (bladder) point

$10 \%$ change due to the $4.5 \mathrm{~mm} \mathrm{~T} \& \mathrm{O}$ reconstruction uncertainty in the caudal direction. ICRU bladder point doses were observed to be more sensitive than bladder $D_{2 c c}$ due to the close proximity of the ovoids.

The impact of T\&O reconstruction-uncertainties was the most at ICRU bladder point doses (Table 3). A $4.5 \mathrm{~mm}$ reconstruction-uncertainty caused $10 \%$ of dosimetric changes to ICRU bladder point doses. A $7.5 \mathrm{~mm}$ reconstruction-uncertainty in the caudal direction resulted in an average $10 \%$ change in ICRU rectal point doses. HR-CTV and point A doses presented relatively small changes, less than $10 \%$ per $\pm 7.5 \mathrm{~mm}$ T\&O reconstruction uncertainties, for either point A plans or MRIG-CBT.

\section{Discussion}

To the best of our knowledge, this is the first study of dosimetric impact due to T\&O applicator displacements for both point A and MRIG-CBT plans. In addition, this is the first study presenting reconstruction uncertainty impact for both point A plans and MRIG-CBT plans. Regarding the impact of T\&O displacements, MRIG-CBT plans demonstrate significantly larger dosimetric changes on sigmoid $(p<0.0005)$, bladder $(p<0.0001)$, HR-CTV $(p<0.0036)$ than those of point A plans. It is mainly due to conformal isodose line target volumes and OARs. However, regardless of MRIG-CBT plans and point A plans, 
Table 3. The simulated dosimetric impacts of T\&O reconstruction-uncertainties for MRIG-CBT (20 plans)

\begin{tabular}{|c|c|c|c|c|c|c|c|c|c|c|c|c|}
\hline & \multicolumn{12}{|c|}{ Simulated dosimetric impact [\%] of T\&O reconstruction-uncertainties for MRIG-CBT plans } \\
\hline & \multicolumn{6}{|c|}{ Caudal tandem shift \& Anterior ovoid shift } & \multicolumn{6}{|c|}{ Cranial tandem shift \& Posterior ovoid shift } \\
\hline & $-10 \mathrm{~mm}$ & $-7.5 \mathrm{~mm}$ & $-6 \mathrm{~mm}$ & $-4.5 \mathrm{~mm}$ & $-3 \mathrm{~mm}$ & $-1.5 \mathrm{~mm}$ & $1.5 \mathrm{~mm}$ & $3 \mathrm{~mm}$ & $4.5 \mathrm{~mm}$ & $6 \mathrm{~mm}$ & $7.5 \mathrm{~mm}$ & $10 \mathrm{~mm}$ \\
\hline $\mathrm{HR}-\mathrm{CTV} \mathrm{D}_{90}[\%]$ & $4 \pm 4$ & $3 \pm 4$ & $3 \pm 3$ & $2 \pm 3$ & $2 \pm 2$ & $1 \pm 1$ & $1 \pm 1$ & $2 \pm 2$ & $3 \pm 3$ & $4 \pm 5$ & $5 \pm 6$ & $8 \pm 8$ \\
\hline HR-CTV D ${ }_{100}[\%]$ & $8 \pm 9$ & $7 \pm 8$ & $6 \pm 6$ & $4 \pm 5$ & $4 \pm 3$ & $2 \pm 2$ & $2 \pm 2$ & $3 \pm 3$ & $4 \pm 4$ & $5 \pm 6$ & $6 \pm 6$ & $10 \pm 9$ \\
\hline Rectum $D_{2 c c}[\%]$ & $8 \pm 7$ & $9 \pm 8$ & $9 \pm 8$ & $7 \pm 6$ & $5 \pm 4$ & $2 \pm 2$ & $1 \pm 1$ & $3 \pm 2$ & $5 \pm 5$ & $7 \pm 5$ & $8 \pm 7$ & $14 \pm 14$ \\
\hline Badder $\mathrm{D}_{2 c \mathrm{c}}[\%]$ & $8 \pm 11$ & $6 \pm 9$ & $5 \pm 7$ & $4 \pm 5$ & $3 \pm 3$ & $2 \pm 2$ & $2 \pm 2$ & $4 \pm 3$ & $6 \pm 5$ & $9 \pm 6$ & $11 \pm 7$ & $16 \pm 11$ \\
\hline Sigmoid $D_{2 c c}[\%]$ & $5 \pm 6$ & $4 \pm 5$ & $4 \pm 4$ & $3 \pm 3$ & $3 \pm 3$ & $2 \pm 3$ & $1 \pm 2$ & $3 \pm 3$ & $4 \pm 6$ & $6 \pm 6$ & $7 \pm 7$ & $11 \pm 9$ \\
\hline Point A left [\%] & $4 \pm 3$ & $3 \pm 2$ & $3 \pm 2$ & $2 \pm 2$ & $2 \pm 1$ & $1 \pm 1$ & $1 \pm 1$ & $2 \pm 2$ & $3 \pm 2$ & $4 \pm 3$ & $5 \pm 4$ & $8 \pm 7$ \\
\hline Point A right [\%] & $4 \pm 3$ & $3 \pm 3$ & $4 \pm 3$ & $3 \pm 2$ & $2 \pm 2$ & $2 \pm 2$ & $1 \pm 2$ & $3 \pm 4$ & $3 \pm 4$ & $4 \pm 5$ & $5 \pm 4$ & $7 \pm 6$ \\
\hline ICRU rectum [\%] & $12 \pm 9$ & $10 \pm 9$ & $8 \pm 8$ & $6 \pm 7$ & $4 \pm 4$ & $2 \pm 2$ & $2 \pm 2$ & $4 \pm 4$ & $6 \pm 6$ & $8 \pm 7$ & $10 \pm 8$ & $13 \pm 11$ \\
\hline ICRU bladder [\%] & $15 \pm 16$ & $12 \pm 13$ & $10 \pm 10$ & $8 \pm 8$ & $7 \pm 5$ & $4 \pm 2$ & $4 \pm 2$ & $7 \pm 4$ & $10 \pm 6$ & $13 \pm 7$ & $16 \pm 9$ & $21 \pm 10$ \\
\hline
\end{tabular}

$<5 \%$

$\geq 5 \%$

$\geq 10 \%$

T\&O -tandem-and-ovoids, HR-CTV - high risk clinical target volume, $D_{90}\left(D_{100}\right)$ - minimum dose covering $90 \%$ (100\%) of the volume, $D_{2 c c}-$ dose of the $2 c c$ of the highest dose, ICRU rectum (bladder) - ICRU report \#38 defined rectal (bladder) point

Table 4. The simulated dosimetric impacts of T\&O reconstruction-uncertainties for conventional planning (20 plans)

\begin{tabular}{|c|c|c|c|c|c|c|c|c|c|c|c|c|}
\hline & \multicolumn{12}{|c|}{ Simulated dosimetric impact [\%] of $T \& O$ reconstruction-uncertainties for conventional point A plans } \\
\hline & \multicolumn{6}{|c|}{ Caudal tandem shift \& Anterior ovoid shift } & \multicolumn{6}{|c|}{ Cranial tandem shift \& Posterior ovoid shift } \\
\hline & $-10 \mathrm{~mm}$ & $-7.5 \mathrm{~mm}$ & $-6 \mathrm{~mm}$ & $-4.5 \mathrm{~mm}$ & $-3 \mathrm{~mm}$ & $-1.5 \mathrm{~mm}$ & $1.5 \mathrm{~mm}$ & $3 \mathrm{~mm}$ & $4.5 \mathrm{~mm}$ & $6 \mathrm{~mm}$ & $7.5 \mathrm{~mm}$ & $10 \mathrm{~mm}$ \\
\hline HR-CTV D $90[\%]$ & $5 \pm 5$ & $4 \pm 3$ & $4 \pm 3$ & $2 \pm 2$ & $2 \pm 1$ & $2 \pm 2$ & $2 \pm 2$ & $4 \pm 4$ & $5 \pm 5$ & $5 \pm 5$ & $6 \pm 5$ & $7 \pm 5$ \\
\hline HR-CTV D ${ }_{100}[\%]$ & $8 \pm 9$ & $6 \pm 5$ & $5 \pm 5$ & $3 \pm 2$ & $3 \pm 2$ & $4 \pm 3$ & $3 \pm 3$ & $5 \pm 5$ & $6 \pm 5$ & $8 \pm 6$ & $9 \pm 7$ & $10 \pm 7$ \\
\hline Rectum $D_{2 c c}[\%]$ & $19 \pm 10$ & $14 \pm 8$ & $13 \pm 8$ & $11 \pm 6$ & $9 \pm 6$ & $5 \pm 4$ & $7 \pm 4$ & $15 \pm 8$ & $23 \pm 11$ & $33 \pm 15$ & $43 \pm 22$ & $65 \pm 32$ \\
\hline Badder $D_{2 c c}[\%]$ & $10 \pm 11$ & $7 \pm 6$ & $6 \pm 5$ & $5 \pm 3$ & $3 \pm 3$ & $3 \pm 2$ & $2 \pm 1$ & $3 \pm 2$ & $4 \pm 2$ & $5 \pm 3$ & $6 \pm 4$ & $8 \pm 6$ \\
\hline Sigmoid $\mathrm{D}_{2 \mathrm{cc}}[\%]$ & $8 \pm 7$ & $7 \pm 6$ & $7 \pm 7$ & $6 \pm 6$ & $5 \pm 5$ & $6 \pm 6$ & $6 \pm 4$ & $8 \pm 6$ & $9 \pm 6$ & $12 \pm 8$ & $12 \pm 10$ & $16 \pm 13$ \\
\hline Point A left [\%] & $1 \pm 1$ & $1 \pm 1$ & $2 \pm 1$ & $1 \pm 2$ & $1 \pm 1$ & $1 \pm 1$ & $1 \pm 1$ & $1 \pm 1$ & $1 \pm 1$ & $2 \pm 2$ & $1 \pm 1$ & $2 \pm 1$ \\
\hline Point A right [\%] & $1 \pm 1$ & $1 \pm 1$ & $1 \pm 1$ & $1 \pm 1$ & $1 \pm 1$ & $1 \pm 1$ & $1 \pm 1$ & $1 \pm 1$ & $1 \pm 1$ & $2 \pm 3$ & $1 \pm 1$ & $1 \pm 1$ \\
\hline ICRU rectum [\%] & $17 \pm 9$ & $12 \pm 7$ & $10 \pm 7$ & $8 \pm 6$ & $6 \pm 5$ & $4 \pm 4$ & $4 \pm 3$ & $9 \pm 6$ & $14 \pm 7$ & $20 \pm 10$ & $25 \pm 13$ & $41 \pm 26$ \\
\hline ICRU bladder [\%] & $23 \pm 23$ & $18 \pm 16$ & $14 \pm 14$ & $11 \pm 10$ & $8 \pm 7$ & $5 \pm 5$ & $3 \pm 3$ & $5 \pm 3$ & $7 \pm 3$ & $9 \pm 4$ & $11 \pm 6$ & $15 \pm 8$ \\
\hline
\end{tabular}

\section{$<5 \% \quad \geq 5 \% \quad \geq 10 \% \quad \geq 15$}

$T \& O$ - tandem-and-ovoids, HR-CTV - high risk clinical target volume, $D_{90}\left(D_{100}\right)$ - minimum dose covering $90 \%(100 \%)$ of the volume, $D_{2 c c}-$ dose of the $2 c c 0$ of the highest dose, ICRU rectum (bladder) - ICRU report \#38 defined rectal (bladder) point

rectal $D_{2 c c}$ changed more than $10 \%$ for $\pm 3 \mathrm{~mm}$ T\&O displacements. Our findings agree with those of Tanderup et al. [8]. They found a mean change of $15 \%$ for the $D_{2 c c}$ values of the rectum and bladder due to $3 \mathrm{~mm}$ cranial-caudal displacement, when T\&R or combined T\&R + needles applicators were used for MRIG-CBT plans. Based upon our findings, $\mathrm{a} \pm 1.5 \mathrm{~mm} \mathrm{~T} \& \mathrm{O}$ displacement allowed all dosimetric metrics to change less than $10 \%$. For the purpose of this study, we simulated motion in both cranial and caudal directions, but in clinical practice, $\mathrm{T} \& \mathrm{O}$ displacement in the caudal direction typically occurs due to movement of the patient anatomy. As yet, no study presents intracavitary applicator displacements due to patient transfer for 3D image scans and their dosimetric impacts on dose-volumetric parameters using 3D images. Gerszten et al. reported an avg. $5 \mathrm{~mm}$ (range, 3 to $12 \mathrm{~mm}$ ) displacement when using an immobilization device (see Fig. 2 in [9]) and 2D radiograph images. It is important to minimize intracavitary applicator displacement when transferring patients. One way to limit patient transfer for 3D image acquisition for image-guided HDR would be to install a CT scanner in the HDR BT room. However, CT provides limited information about macroscopic tumor volume for cervical cancer that MRI is able to provide [5]. Another possible solution is to utilize one of the commercial immobilization systems such as those available from Zephyr patient transport system (DIACOR Inc., Salt Lake City, Utah, USA) using air-bearing techniques or MedTrak (MedTrak, LLC, Madison, Wisconsin, USA) that uses ceiling rails. Those systems are expected to reduce applicator displacement due to patient transfer. However, none of these support devices address applicator immobilization with respect to the patient. Our T\&O support device was securely attached to the patient, 
allowing it to move as the patient does. In contrast to this, in some immobilization systems the immobilization device (see Fig. 2 in [9]) is secured to a transfer-board or a table, which can cause damage like uterine perforation due to even small patient movements. Applicator displacement can occur due to one or many reasons including anatomy changes due to insufficient anesthesia, suboptimal vaginal packing, and vibrations from the transfer table while in motion between rooms. Thus, the use of an applicator immobilizing device with respect to a patient is recommended even when a commercial immobilization system is in place. Even when using immobilization systems, applicator displacement must be carefully monitored when a patient is moved to different rooms for MRI or CT imaging. Studies for a robust immobilization system that limits motion, yet is still cost effective are expected to proliferate as 3D image-guided BT is more widely practiced in clinics.

Recently, planning uncertainties on 3D image guided BT have been widely explored in the literature. An sample of some of the reports include: the impact of uncertainties on planning and prescription [25], inter- and intra-fractional anatomical variations [26] and their dosimetric impact [27], especially for pulse-dose-rate (PDR) $\mathrm{BT}$, and critical structure movement between planning MRI and secondary MRI before HDR [28] treatment. The overall $\mathrm{D}_{2 \mathrm{cc}}$ value of multiple BT fractions is obtained under the assumption that the approximate $\mathrm{D}_{2 \mathrm{cc}}$ location remains the same through multiple fractions. Jamema et al. [29] evaluated inter-application variation of doses and spatial location of OAR $\mathrm{D}_{2 \mathrm{cc}}$ and found that the $\mathrm{D}_{2 \mathrm{cc}}$ locations were quite stable for bladder and rectum. However, significant topographical changes were reported for the sigmoid [29]. For the bladder, the current simple DVH parameter addition was also validated as compared to the overall DVH through deformable image registration (DIR) by Andersen et al. [30]. They found a current $\mathrm{D}_{2 c c}$ addition for the bladder provides a good estimate, whereas $D_{0.1 c c}$ is less robust when compared to the values obtained using DIR. In relation to target volume delineation uncertainties, Petrič et al. found 0.76 (76\% agreement) and 0.72 for HR-CTV for its simultaneous truth and performance level estimation, and expert consensus, respectively [31]. HR-CTV was found to be more robust than gross target volume and intermediate risk CTV. The dosimetric impact of delineation uncertainty for HR-CTV and OARs were quantified by Hellebust et al. [32]. They found that interobserver delineation variability caused \pm $5 G y_{\alpha / \beta}=10$ of equivalent dose in $2 \mathrm{~Gy}$ fraction (EQD2) for HR-CTV $D_{90}$ and $\pm 2-3$ of EQD2 for OAR $D_{2 c c}$. Treatment planning technique variations among four T\&O user institutions were compared for point A plans, T\&O MRIGCBT plans, and T\&O + needles MRIG-CBT plans [33]. Results indicated that adding interstitial needles to MRIG-CBT plans resulted in higher tumor coverage, but increased the variety in dose distributions between the institutions [33]. The uncertainties of performing two HDR fractions within one applicator implant were reported by Lang et al. [34]. They concluded that geometric differences between applicator, target, and OAR resulted in an overall dosimetric change $\left( \pm 1.2 \mathrm{G} y_{\alpha / \beta}=10 \mathrm{EQD} 2\right.$ of HR-CTV $D_{90}$ and $\pm 0.7-1.1 G y_{\alpha / \beta}=3$ EQD2 for OAR $D_{2 c c}$ ) that could be of minor relevance in regard to clinical dose volume constraints.

To account for geometric uncertainties of applicator reconstruction or displacements, Tanderup et al. [8,24] proposed a margin in the cranial direction only along a tandem (see Fig. 1 in [24]). Current 3D-imaging guidelines for GYN cancer [2-4,18] do not have margins to account for the uncertainties due to applicator displacements and/or an applicator reconstruction inaccuracy $[4,8]$. As highlighted by Tanderup et al. [24], the general planning target volume (PTV) concept should not be used to compensate for the uncertainties in $3 \mathrm{D}$ image guided brachytherapy (see Fig. 2 in [24]). They recommended not creating a general PTV margin, especially in the lateral and anterior-posterior directions as that could lead to a significant overall dose escalation: $\sim 8 \% / \mathrm{mm}$ within the margin applied. Dose prescription or normalization to PTV was also strongly discouraged in the study [24].

\section{Conclusions}

Regarding the dosimetric impact of $\mathrm{T} \& \mathrm{O}$ displacement, rectal $D_{2 c c}$ values were observed as being most sensitive to change due to the $\mathrm{T} \& \mathrm{O}$ displacement among all dosimetric metrics regardless of point $\mathrm{A}$ $(p<0.013)$ or MRIG-CBT plans $(p<0.0277)$. To avoid greater than $10 \%$ changes, no more than $\pm 1.5 \mathrm{~mm}$ $\mathrm{T} \& \mathrm{O}$ displacements were allowed for both point $\mathrm{A}$ and MRIG-CBT plans. The dosimetric impact of simulated T\&O displacements on sigmoid $(p<0.0005)$, bladder $(p<0.0001)$, HR-CTV $(p<0.0036)$, and point A $(p<0.0015)$ were significantly larger in MRIG-CBT plans than point A plans. Regarding the dosimetric impact of $\mathrm{T} \& \mathrm{O}$ reconstruction-uncertainties, less than a $\pm 1.5 \mathrm{~mm}$ reconstruction-uncertainties was required in order to avoid a greater than $10 \%$ dosimetric change to point A plans.

\section{Acknowledgements}

This work was partly supported by a research collaboration grant from Varian Medical System, Inc.

\section{References}

1. Viswanathan AN, Erickson BA. Three-dimensional imaging in gynecologic brachytherapy: a survey of the American Brachytherapy Society. Int J Radiat Oncol Biol Phys 2010; 76: 104-109.

2. Haie-Meder C, Pötter R, Van Limbergen E et al. Recommendations from gynaecological (GYN) GEC-ESTRO Working Group (I): concepts and terms in 3D image based 3D treatment planning in cervix cancer brachytherapy with emphasis on MRI assessment of GTV and CTV. Radiother Oncol 2005; 74: 235-245.

3. Pötter R, Haie-Meder C, Van Limbergen E et al. Recommendations from gynaecological (GYN) GEC ESTRO working group (II): concepts and terms in 3D image-based treatment planning in cervix cancer brachytherapy-3D dose volume parameters and aspects of 3D image-based anatomy, radiation physics, radiobiology. Radiother Oncol 2006; 78: 67-77. 
4. Hellebust TP, Kirisits C, Berger D et al. Recommendations from gynaecological (GYN) GEC-ESTRO working group: considerations and pitfalls in commissioning and applicator reconstruction in 3D image-based treatment planning of cervix cancer brachytherapy. Radiother Oncol 2010; 96: 153-160.

5. Viswanathan AN, Dimopoulos J, Kirisits C et al. Computed tomography versus magnetic resonance imaging-based contouring in cervical cancer brachytherapy: results of a prospective trial and preliminary guidelines for standardized contours. Int J Radiat Oncol Biol Phys 2007; 68: 491-498.

6. Anderson J, Huang Y, Kim Y. Dosimetric impact of point A definition on high-dose-rate brachytherapy for cervical cancer: evaluations on conventional point A and MRI-guided, conformal plans. J Contemp Brachytherapy 2012; 4: 241-246.

7. Anderson J, Xia J, Flynn RT et al. High resolution (3 Tesla) MRI-guided conformal brachytherapy for cervical cancer: consequences of different high-risk CTV sizes. J Contemp Brachytherapy 2013; 5: 101-109.

8. Tanderup K, Hellebust TP, Lang S et al. Consequences of random and systematic reconstruction uncertainties in 3D image based brachytherapy in cervical cancer. Radiother Oncol 2008; 89: 156-163.

9. Gerszten K, Paul C, Kin G et al. High dose rate tandem and ring applicator movement with patient transfer from simulation to treatment room. J Brachytherapy Int 1998; 14: 15-20.

10. Xia J, Waldron T, Kim YA. Real-Time Applicator Position Monitoring System (RAPS) for High-Dose-Rate Intracavitary Brachytherapy. Med Phys 2013; 40: 465.

11. Perez-Calatayud J, Kuipers F, Ballester F et al. Exclusive MRI-based tandem and colpostats reconstruction in gynaecological brachytherapy treatment planning. Radiother Oncol 2009; 91: 181-186.

12. De Leeuw AA, Moerland MA, Nomden C et al. Applicator reconstruction and applicator shifts in 3D MR-based PDR brachytherapy of cervical cancer. Radiother Oncol 2009; 93 : 341-346.

13. Haack S, Nielsen SK, Lindegaard JC et al. Applicator reconstruction in MRI 3D image-based dose planning of brachytherapy for cervical cancer. Radiother Oncol 2009; 91: 187-193.

14. Petit S, Wielopolski P, Rijnsdorp R et al. MR guided applicator reconstruction for brachytherapy of cervical cancer using the novel titanium Rotterdam applicator. Radiother Oncol 2013; 107: 88-92

15. Berger D, Dimopoulos J, Pötter R et al. Direct reconstruction of the Vienna applicator on MR images. Radiother Oncol 2009; 93: 347-351.

16. Schindel J, Muruganandham M, Pigge FC et al. Magnetic resonance imaging (MRI) markers for MRI-guided highdose-rate brachytherapy: novel marker-flange for cervical cancer and marker catheters for prostate cancer. Int J Radiat Oncol Biol Phys 2013; 86: 387-393.

17. Viswanathan AN, Thomadsen B and Committee ABS. CCR American Brachytherapy Society consensus guidelines for locally advanced carcinoma of the cervix. Part I: general principles. Brachytherapy 2012; 11: 33-46.

18. Viswanathan AN, Beriwal S, De Los Santos JF et al. American Brachytherapy Society consensus guidelines for locally advanced carcinoma of the cervix. Part II: high-dose-rate brachytherapy. Brachytherapy 2012; 11: 47-52.

19. Kim Y, Muruganandham M, Modrick JM et al. Evaluation of artifacts and distortions of titanium applicators on 3.0-Tesla MRI: feasibility of titanium applicators in MRI-guided brachytherapy for gynecological cancer. Int J Radiat Oncol Biol Phys 2011; 80: 947-955.

20. Anderson J, Huang Y, Kim Y. Dosimetric impact of point A definition on high-dose-rate brachytherapy for cervical can- cer: evaluations on conventional point A and MRI-guided, conformal plans. J Contemp Brachytherapy 2012; 4: 241-246.

21. Rockey WM, Bhatia SK, Jacobson GM et al. The dosimetric impact of vaginal balloon-packing on intracavitary highdose-rate brachytherapy for gynecological cancer. J Contemp Brachytherapy 2013; 5: 17-22.

22. Sun W, Bhatia SK, Jacobson GM et al. The Changes of Target Volumes Through High Dose Rate Brachytherapy for Cervical Cancer When evaluated on High Resolution (3.0) MRI. Practical Radiation Oncology 2012; 2: e101-e106.

23. Kim Y, Modrick JM, Bayouth JE et al. Logistics of tandem and ovoids HDR plan optimization in MRI-guided brachytherapy for cervical cancer: comparisons of standard, graphical, and inverse optimization. Brachytherapy 2009; 8: 121-122.

24. Tanderup K, Pötter R, Lindegaard JC et al. PTV margins should not be used to compensate for uncertainties in 3D image guided intracavitary brachytherapy. Radiother Oncol 2010; 97: 495-500.

25. Tanderup K, Nesvacil N, Pötter R et al. Uncertainties in image guided adaptive cervix cancer brachytherapy: impact on planning and prescription. Radiother Oncol 2013; 107: 1-5.

26. Nesvacil N, Tanderup K, Hellebust TP et al. A multicentre comparison of the dosimetric impact of inter- and intra-fractional anatomical variations in fractionated cervix cancer brachytherapy. Radiother Oncol 2013; 107: 20-25.

27. Morgia M, Cuartero J, Walsh L et al. Tumor and normal tissue dosimetry changes during MR-guided pulsed-dose-rate (PDR) brachytherapy for cervical cancer. Radiother Oncol 2013; 107: 46-51.

28. Anderson C, Lowe G, Wills R et al. Critical structure movement in cervix brachytherapy. Radiother Oncol 2013; 107: 39-45.

29. Jamema SV, Mahantshetty U, Tanderup K et al. Inter-application variation of dose and spatial location of $\mathrm{D}(2 \mathrm{~cm}(3))$ volumes of OARs during MR image based cervix brachytherapy. Radiother Oncol 2013; 107: 58-62.

30. Andersen ES, Noe KO, Sorensen TS et al. Simple DVH parameter addition as compared to deformable registration for bladder dose accumulation in cervix cancer brachytherapy. Radiother Oncol 2013; 107: 52-57.

31. Petric P, Hudej R, Rogelj P et al. Uncertainties of target volume delineation in MRI guided adaptive brachytherapy of cervix cancer: a multi-institutional study. Radiother Oncol 2013; 107: 6-12

32. Hellebust TP, Tanderup K, Lervag C et al. Dosimetric impact of interobserver variability in MRI-based delineation for cervical cancer brachytherapy. Radiother Oncol 2013; 107: 13-19.

33. Nomden CN, de Leeuw AA, Van Limbergen E et al. Multicentre treatment planning study of MRI-guided brachytherapy for cervical cancer: comparison between tandem-ovoid applicator users. Radiother Oncol 2013; 107: 82-87.

34. Lang S, Nesvacil N, Kirisits $C$ et al. Uncertainty analysis for 3D image-based cervix cancer brachytherapy by repetitive MR imaging: assessment of DVH-variations between two HDR fractions within one applicator insertion and their clinical relevance. Radiother Oncol 2013; 107: 26-31. 